\title{
A Review of Pharmacotherapy in the Treatment of Urinary Disorders
}

\author{
Dr Manoj G Tyagi \\ Department of Pharmacology, KM Medical College, Mathura, Uttar Pradesh \\ *Author for Correspondence: Dr Manoj G Tyagi; tyagi243@yahoo.co.in
}

Received 26 May 2019;

Accepted 14 June 2019;

Published 17 June 2019

\begin{abstract}
Urinary tract infections and other related pathophysiological conditions are a plethora of ailments affecting mankind. Ailments that are as common as the urinary tract infection, uropathy, incontinence, calculi and many other disorders. With the advancement of research into newer and more potent drugs with better efficacy and lesser side effects, treatment is becoming more specific and efficacious. This review examines the recent development in the treatment of these disorders and impressive progress in the pharmacotherapy, it also gives an overview of current and future prospects of drugs for bladder disorders.
\end{abstract}

Keywords: Bladder, incontinence, urinary, anti-cholinergic, disorder

\section{Introduction}

Urinary tract disorders are one of the common disorders of human beings. The Urinary tract system comprises of the ureters, bladders and urethra and these are the key excretory system for not just metabolic by products, toxins and xenobiotics and always under a lot of pressure and stress ${ }^{[1]}$. This sometimes leads to infections, wear and tear and pathophysiological disorders. The most common of course amongst these is the urinary tract infections, uropathy, incontinence, calculi and bladder incontinence and retention. Recent research has focused on anti-cholinergics and Beta 3 agonists. There is a growing and pervasive need for pharmacologic therapies that are efficacious, safe, and tolerable ${ }^{[2]}$. Efforts in the past few years have identified potential new targets in addition to combination therapies or adding a non-pharmacological intervention or both. This article examines the current treatment and recent progress in the treatment of these disorders.

\section{The Urinary System}

The urinary system, also known as the renal system, produces, stores and eliminates urine, the fluid waste excreted by the kidneys. The kidneys make urine by filtering wastes and extra water from blood. Urine travels from the kidneys through two thin tubes called ureters and fills the bladder [Fig.1]. The urinary system works with the lungs, skin and intestines to maintain the balance of chemicals and water in the body. Adults eliminate about 27 to 68 fluid ounces (800 to 2,000 milliliters) per day based on typical daily fluid intake of 68 ounces ( 2 liters). From the kidneys, urine travels down two thin tubes, called ureters, to the urinary bladder. The ureters are about 8 to 10 inches long ( 20 to 25 centimeters).The bladder stores urine until the brain signals the bladder that the person is ready to void $\mathrm{it}^{[3]}$. A normal, healthy bladder can hold up to 16 ounces i.e almost half a liter of urine comfortably for two to five hours.

\section{Bladder and Sphincter Muscles}

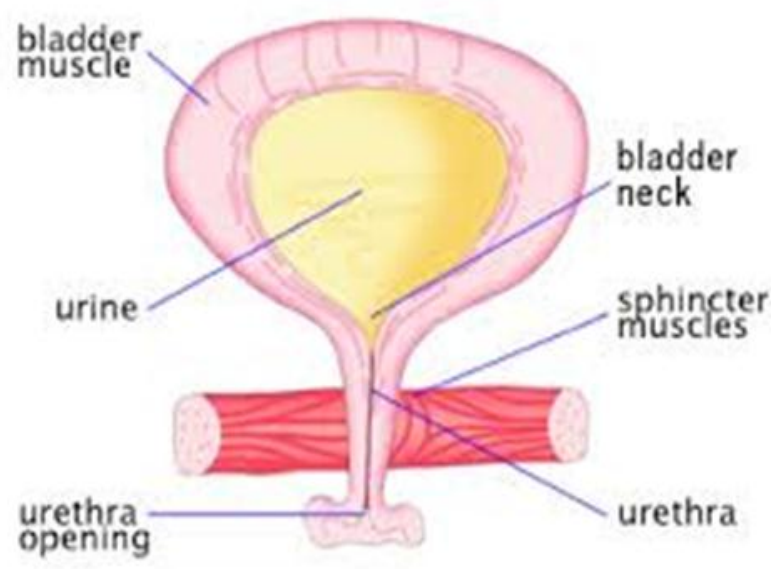

Fig.1 Anatomical structure of Urinary Bladder

To prevent leakage, circular muscles called sphincters close tightly around the opening of the bladder into the urethra, the tube that allows urine to pass outside the body ${ }^{[4]}$. The only difference between the female and male urinary system is the length of the urethra. In females, the urethra is about 1.5 to 2 inches long (3.8 to $5.1 \mathrm{~cm})$. In males, it is about 8 inches $(20 \mathrm{~cm})$ long.

\section{Diseases of the renal system}


Urinary tract infections (UTIs) occur when bacterial infection affects the urinary tract; they can affect the urethra, bladder or even the kidneys. While UTIs are more common in women, they can occur in men. UTIs are typically treated with antibiotics. Some of the common drugs treating UTI are Cephalosporins, Fosfomycin and Sulphonamides. New Drug for UTI: The drug, Vabomere, contains the antibiotic meropenem and the novel beta-lactamase inhibitor vaborbactam. Vabomere was developed to treat serious gram-negative infections, including cUTI, caused by carbapenemresistant Enterobacteriaceae (CRE). CRE have been classified as an urgent health threat by the US Centers for Disease Control and Prevention and a priority pathogen by the World Health Organization.

\section{Urinary Incontinence}

The pathophysiology underlying urge induced urinary incontinence (UUI) is complex and based on altered physiological mechanisms controlling bladder storage and emptying. Causal factors have been focused on myogenic, neurogenic, and recently shown to be due to sensory dysfunction. Stress induced urinary incontinence (SUI) is urinary leakage due to increased abdominal pressure caused by sneezing, coughing, exercise, lifting, and position change.Incontinence is another common disease of the urinary system. "The most common bladder problems I see in my practice in women are frequent urges to urinate and leakage of urine," said Dr S. Adam Ramin, urologic surgeon and founder of Urology Cancer Specialists in Los Angeles. "The most common bladder problems in men are frequent urination at nights and incomplete bladder emptying. This is usually due to an enlarged prostate causing obstruction of bladder emptying ${ }^{[5]}$.

\section{Potassium channel openers for Medical expulsion therapy}

Potassium channel openers affect Kv type channels have been advocated for removal of renal stones/calculi stuck in the ureter and outer portion of the calyx as they tend to relax and cause the removal of these materials from the urinary tract. In this regards drugs like Retigabine and Flupirtine have been tested and found to be effective ${ }^{[6,7]}$. Flupirtine has additional NMDA receptor antagonistic properties.

Interstitial cystitis (IC), also called painful bladder syndrome, is a chronic bladder condition, primarily in women, that causes bladder pressure and pain and, sometimes, pelvic pain to varying degrees, according to the Mayo Clinic. Botulinum toxin A has been found to give relief for several months ${ }^{[8]}$. It can cause bladder scarring, and can make the bladder less elastic. While the cause isn't known, many people with the condition also have a defect in their epithelium, the protective lining of the bladder.

On the other hand, Prostatitis is a swelling of the prostate gland and, therefore, can only occur in men. Often caused by advanced age, symptoms include urinary urgency and frequency, pelvic pain and pain during urination and treatable with chemotherapeutic agents like fluoroquinolones and doxycycline.

\section{Novel drug delivery systems for treating bladder disorders:}

The goal of targeted delivery drugs is to transport proper amounts of drugs to the desirable sites while minimizing unwanted side effects on other tissues. Several strategies have been developed within the last decade. Various drug formulations, routes of drug administration, as well as devices for drug delivery and targets have been described. The bladder is an ideal target for local drug delivery as it is easily accessed through the urethra. Intravesical drug delivery (IDD) is however hindered by continuous filling of the bladder with urine, the low permeability of the bladder permeability barrier (BPB), and the washout during voiding. Hence, serial drug instillations are often required.

In the last decade, novel DDSs have been devised with the aim to enhance the therapeutic efficacy and reduce costs linked to the discovery of new drugs. Nanotechnology has revolutionized the field of drug delivery and represents a great potential for the future. This will require interdisciplinary teams including clinicians, biologists, engineers, and pharmaceutical scientists ${ }^{[9]}$.

\section{Drugs for Bladder disorders}

Intravesical botulinum toxin A (BoNT/A) preventsacetylcholine release at the neuromuscular junction, resulting in temporary chemodenervation and muscle relaxation for up to 6 months. The technique is to place multiple injections under cystoscopic guidance directly into the detrusor.

Complete continence can be achieved in $40-80 \%$ of patients and bladder capacity improved by $56 \%$ for up to 6 months. Maximal benefit is between 2 and 6 weeks, maintained over 6 months. The injections can be repeated. Oxybutinin can be started at $2.5 \mathrm{mg}$ twice per day. The morning dose can be increased or a lunchtime dose added depending on severity and timing of symptoms. The maximum dose is $5 \mathrm{mg}$ three times per day. High doses are often not well tolerated due to dry mouth, gritty eyes, exacerbation of gastrointestinal reflux and constipation ${ }^{[10-11]}$. For Interstitial cystitis, various oral treatments (tablets, capsules or syrup) are used for IC. The advantage of oral treatment is that it is easy to administer and non-invasive. Amitriptyline an antidepressant drug is currently recommended as a standard first-line oral treatment for the pain of IC, is generic, inexpensive and widely available. It should be taken at dinner in the evening and will help the patient to sleep. By taking at dinner time rather than just before going to sleep, morning hangover can be avoided. This drug is believed to have multiple qualities including pain relief, anticholinergic effects and neuromodulation. It is usual to start with a low dosage (10 mg or less) and gradually increase to optimum toleration level (with minimum side effects) for the individual patient (up to maximum $75 \mathrm{mg}$. Corticosteroids (e.g. hydrocortisone, prednisolone and dexamethasone) are a group of drugs with a strong antiinflammatory effect. Although prolonged use can lead to the risk of serious side-effects such as osteoporosis and lowered resistance to infection, these drugs can nevertheless be of great benefit to some IC patients. Montelukast (e.g. Singulair $\left.{ }^{\circledR}\right)$, a medication used to treat asthma, inhibits the release of leukotrienes from mast cells and other cell types and thereby prevents inflammation. Quercetin: this bioflavinoid is an alternative strategy for the treatment of IC. It is believed to have anti-inflammatory effects and to inhibit the activation of mast-cells. Has shown promising results and is available over the counter. IP 751 (ajulemic acid): an experimental anti-inflammatory, analgesic drug currently being developed for various specific conditions including IC. IP 751 is a non-psychoactive synthetic cannabinoid that appears to suppress inflammation and pain. Unlike many NSAIDs, IP 751 does not appear to cause gastrointestinal ulceration. Pentosan polysulfate sodium (PPS) PPS is a heparinoid drug. One of the hypotheses 
concerning the causes of IC is the existence of a defect in the glycosaminoglycan (GAG) layer that acts as a protective lining for the wall of the bladder. It is believed that PPS temporarily repairs this defect, creating a synthetic layer that protects the underlying bladder wall from being attacked by irritant elements in the urine. Desmopressin is a man-made form of a natural hormone that your body makes. The pituitary hormone, called antidiuretic hormone $(\mathrm{ADH})$ or vasopressin, directs the kidneys to make less urine. The urine is therefore more concentrated. Desmopressin is not usually prescribed for adult women with overactive bladder or stress incontinence. It is more often used to treat bedwetting in children $^{[12]}$.

\section{Drugs for Urinary Incontinence:}

A) Anti-cholinergic drugs: The drugs listed for urinary incontinence are oxybutynin, Bethanechol, darifenacin, solifenacin, tolterodine, festoterodine. B) Beta 3 agonists: Mirabegron, Solabegron and Vibegron.

\section{Beta 3 Agonists}

The presence of $\beta$-adrenoceptors in rat and human bladders at the mRNA level has been studied using Northern blots, PCR, and in situ hybridization. Studies in the human bladder have detected mRNA for all three $\beta$-adrenoceptor subtypes ${ }^{[12-16]}$ [Fig.2]. Based on quantitative PCR experiments, it appears that the $\beta 3$-adrenoceptor accounts for more than $95 \%$ of all $\beta$-adrenoceptor mRNA in the human bladder ${ }^{[17]}$. The presence of $\beta 3$-adrenoceptors mRNA in the human detrusor has also been confirmed by in situ hybridization studies $^{[18]}$. The identification of $\beta$-adrenoceptors at the protein level is typically based on receptor binding studies with radioligands ${ }^{[19]}$. The 3 most common Beta 3 agonists are namely, Mirabegron, Solabegron and Vibegron. These agents are used in patients with overactive bladder disorders (OAB). Basically they prevent micturition by relaxing the urinary bladder. These agents also cause lipolysis by acting on beta 3 receptors in adipose tissues. According to a study by Cao et al the beta 3 receptors also interact with $\mathrm{Src}$ or cytosolic tyrosine kinases ${ }^{[20]}$. These drugs are available in the market for the treatment of $\mathrm{OAB}$.

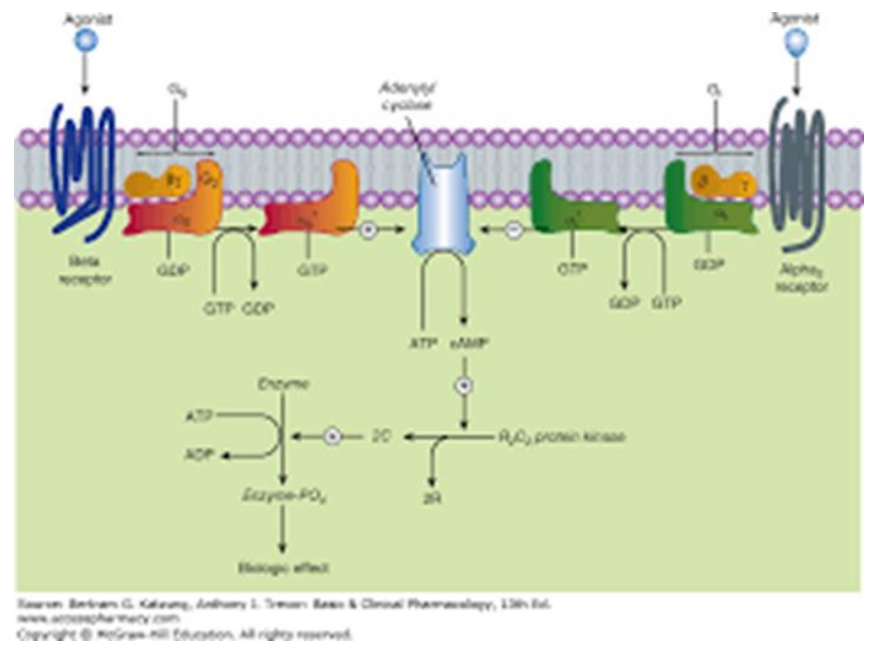

Fig.2 Adrenoceptor mediated signal transduction

\section{Anti-cholinergic drugs}

OAB can be managed with bladder and behavioral training, biofeedback, electric stimulation, pharmacological treatments, or with a combination of these therapies. However, antimuscarinic agents (muscarinic antagonists), such as oxybutynin, propiverine, tolterodine, trospium, darifenacin, solifenacin, imidafenacin, and fesoterodine are currently the first-line pharmacotherapy for $\mathrm{OAB}^{[21-22]}$. Anti-muscarinic drugs were developed to target the main pathway controlling detrusor contraction in which acetylcholine released from parasympathetic nerves activated muscarinic receptors. As aforementioned, of the 5 subtypes of muscarinic receptors, M2 and M3 subtypes are the main subtypes found in the bladder. An M3 receptor directly mediates detrusor contraction. 1) Tolterodine is a non specific competitive muscarinic receptor antagonist $\mathrm{OAB}$. Tolterodine has high specificity for muscarinic receptors in the urinary bladder. It is available as $2 \mathrm{mg}$ tablets twice daily. Tolterodine has been documented to cause mean decrease urge in continence episodes by $50 \%$. 2) Darifenacin HCl: It is an extended release product that elicits competitive muscarinic receptor antagonistic activity. It has high affinity for M3 receptor hence it reduces bladder smooth muscle contractions and reduction in adverse events related to muscarinic blockade. Darifenacin is given as once daily dosing at recommended doses of $7.5 \mathrm{mg}$ to $15 \mathrm{mg}$ per day. 3) Solifenacin: This drug is a new competitive muscarinic antagonist for the treatment of $\mathrm{OAB}$ with symptoms of urgency, urge incontinence and urinary frequency. It shares a similar muscarinic receptor affinity as oxybutynin and available as $5 \mathrm{mg}$ and $10 \mathrm{mg}$ daily ${ }^{[23]}$.4) Oxybutynin: It has anti-cholinergic and direct smooth muscle relaxant effect on the urinary bladder alongwith local anesthetic effect on irritable bladder. The human detrusor muscles have M2 and M3 muscarinic receptors. Oxybutynin has greater affinity for M3 receptor. Oxybutynin increases bladder size, decreases frequency of symptoms and delays initial to void. Oxybutynin is available as extended release, immediate release and transdermal forms in doses of $7.5 \mathrm{mg}$ to $15 \mathrm{mg}$. Oxybutynin transdermal delivery system offers a twice weekly and better patient compliance and tolerability ${ }^{[24-25]}$.

\section{Conclusion}

The pharmacological treatment of urinary bladder disorders is an area of intensive research. New drugs for UTI, Interstital cystitis and $\mathrm{OAB}$ are being introduced in the market. Added to this is the new dimension of targeted drug delivery to the urinary bladder. Thus pharmacotherapy is still the best bet to treat many of the urinary disorders and it appears that even monocolonal antibodies are being introduced. This review has thereby focused on contemporary and novel drugs to treat these disorders.

\section{References}

[1] M G Tyagi.Urinary Bladder Contractility: A Relationship with Central Nervous Activity and Sleep.Current Urology 2(4):217-21

[2] de Groat WC, Yoshimura N. Pharmacology of the lower urinary tract. Annu Rev Pharmacol Toxicol. 2001;41:691-721.

[3] K A Zimmermann, Live Science Contributor | Urinary System: Facts, Functions \& Diseases, March 6, 2018

[4] Yoshimura N, Chancellor MB. Neurophysiology of lower urinary tract function and dysfunction. Rev Urol. 2003;5 (suppl 8):S3-S10.

[5] Sellers DJ, McKay N. Developments in the pharmacotherapy of the overactive bladder. Curr Opin Urol. 2007;17(4):223-230. 
[6] Manoj G Tyagi, GirishS. Naik, John Premendran, Usha H Shah, Kalpana Ernest, Subramanium Kannan. KCNQ potassium channel expression in the caprine ureter and inhibition ofthecontractility of isolated distal caprine ureter by KCNQ channel opener Retigabine.Biomedical Pharm.Res. Volume 6, Issue 5: September-October: 2017, 10-15

[7] Girish S Naik, Rohit Kodagali, Manoj G Tyagi, Kalpana Ernest, Margaret Shanthi, Sumith K Mathew, andJacob Peedicayil.Inhibition of Spontaneous Contractility of Isolated Caprine Ureter by Flupirtine. Int J Appl Basic Med Res. 2018 Apr-Jun; 8(2): 116-119.

[8] L Vas,M Pattanik, V Titarmore. Treatment of interstitial cystitis/ painful bladder syndrome as a neuropathic pain condition. Ind.J.Urol. 2014, 30(3)-350-353

[9] Martino Maria Zacchè,Sushma Srikrishna,Linda Cardozo.Novel targeted bladder drug-delivery systems: a review. Research and Reports in Urology 2015:7 169178

[10] Anger JT, Weinberg A, Suttorp MJ, Litwin MS, Shekelle PG. Outcomes of intravesical botulinum toxin for idiopathic overactive bladder symptoms: a systematic review of the literature. J Urol.2010;183:2258-64.

[11] Karsenty G, Denys P, Amarenco G, De Seze M, Gamé X, Haab F, Kerdraon J, Perrouin-Verbe B, Ruffion A, Saussine C, Soler JM, Schurch B, Chartier-Kastler E. Botulinum toxin A (Botox) intradetrusor injections in adults with neurogenic detrusor overactivity/neurogenic overactive bladder: a systematic literature review. Eur Urol. 2008;53:275-87.

[12] A Chauhan. Drug therapy for urinary disorders. WWW.Fogsi.org.72-78

[13] Koike K, Takayanagi I. Beta3-adrenoceptor mechanisms in guinea-pig taenia caecum. Life Sci. 1998;62(1718):1503-1507.

[14] Nagatomo T, Koike K. Recent advances in structure, binding sites with ligands and pharmacological function of beta-adrenoceptors obtained by molecular biology and molecular modeling. Life Sci. 2000;66(25):2419-2426.

[15] Johnson M. The beta-adrenoceptor. Am J Respir Crit Care Med. 1998;158(5, pt 3):S146-153.

[16] Igawa Y, Yamazaki Y, Takeda H, Hayakawa K, Akahane M, Ajisawa Y, et al. Functional and molecular biological evidence for a possible beta3-adrenoceptor in the human detrusor muscle. $\mathrm{Br} \mathrm{J}$ Pharmacol. 1999;126(3):819-825.

[17] Takeda M, Obara K, Mizusawa T, Tomita Y, Arai K, Tsutsui $\mathrm{T}$, et al. Evidence for beta3-adrenoceptor subtypes in relaxation of the human urinary bladder detrusor: analysis by molecular biological and pharmacological methods. J Pharmacol Exp Ther. 1999;288(3):1367-1373.

[18] Fujimura T, Tamura K, Tsutsumi T, Yamamoto T, Nakamura K, Koibuchi Y, et al. Expression and possible functional role of the beta3-adrenoceptor in human and rat detrusor muscle. J Urol. 1999;161(2):680-685.

[19] Motoaki Saito, Fotios Dimitriadis, Fumiya Ohmasa, Seiya Inoue, Keisuke Satoh. A $\beta 3$ Agonist, Mirabegron for the Treatment of Overactive Bladder. UroToday Int J. 2011 Dec;4(6):

[20] Cao W, Lutteral LM, Medvedev AV, Pierce KL, Daniel KW, Dixon TM, Lefkowitz RJ, Collins S .Direct binding of activated c-src to the beta 3 adrenergic receptor is required for MAP kinase activation. J Biol. Chem.2000; 275 (49): 38131-4

[21] Kuteesa W, Moore K. Anticholinergic drugs for overactive bladder. Australian Prescriber 2006; 29:22-4.

[22] Chapple CR, Khullar V, Gabriel Z, Muston D, Bitoun CE, Weinstein D. The effects of antimuscarinic treatments in overactive bladder: an update of a systematic review and meta-analysis. Eur Urol. 2008;54(3):543-562

[23] Fetscher C, Fleichman M, Schmidt M, Krege S, Michel MC. M(3) muscarinic receptors mediate contraction of human urinary bladder. $\mathrm{Br} \mathrm{J}$ Pharmacol. 2002;136(5):641-643.

[24] Chess-Williams R, Chapple CR, Yamanishi T, Yasuda $\mathrm{K}$, Sellers DJ. The minor population of M3-receptors mediate contraction of human detrusor muscle in vitro. $\mathrm{J}$ Auton Pharmacol. 2001;21(5-6):243-248.

[25] Andersson KE, Chapple CR. Pharmacological treatment of urinary incontinence. In: Incontinence 4th Edition 2009. Editors; Abrams P, Cardozo L,Khoury S, Wein A (International Continence Society) Health Publication Ltd. 